\begin{tabular}{|c|l|}
\hline Title & Two-Stage Kondo Effect in a Quantum Dot at a High Magnetic Field \\
\hline Author(s) & $\begin{array}{l}\text { van der Wiel, W. G.; De Franceschi, S.; Elzerman, J. M.; Tarucha, S.; Kouw enhoven, L. P.; Motohisa, J.; Nakaji ima, F.; } \\
\text { Fukui, T. }\end{array}$ \\
\hline Citation & $\begin{array}{l}\text { Physical Review Letters, 88(12), 126803 } \\
\text { https://doi.org/10.1103/PhysRevLett.88.126803 }\end{array}$ \\
\hline Issue Date & 2002-03-25 \\
\hline Doc URL & http://hdl.handle.net/2115/5523 \\
\hline Rights & Copyright $\odot 2002$ A merican Physical Society \\
\hline Type & article \\
\hline File Information & PRL88-12.pdf \\
\hline
\end{tabular}

Instructions for use 


\title{
Two-Stage Kondo Effect in a Quantum Dot at a High Magnetic Field
}

\author{
W. G. van der Wiel, ${ }^{1}$ S. De Franceschi, ${ }^{1}$ J. M. Elzerman, ${ }^{1}$ S. Tarucha, ${ }^{2}$ and L. P. Kouwenhoven ${ }^{1}$ \\ ${ }^{1}$ Department of Applied Physics, DIMES, and ERATO Mesoscopic Correlation Project, Delft University of Technology, \\ P.O. Box 5046, 2600 GA Delft, The Netherlands \\ ${ }^{2}$ ERATO Mesoscopic Correlation Project, University of Tokyo, 7-3-1, Hongo, Bunkyo-ku, Tokyo 113-0033, Japan \\ J. Motohisa, F. Nakajima, and T. Fukui \\ Research Center for Integrated Quantum Electronics, Hokkaido University, North 13, West 8, Sapporo 060-8628, Japan
} (Received 19 October 2001; published 7 March 2002)

\begin{abstract}
We report a strong Kondo effect (Kondo temperature $\sim 4 \mathrm{~K}$ ) at high magnetic field in a selective area growth semiconductor quantum dot. The Kondo effect is ascribed to a singlet-triplet transition in the ground state of the dot. At the transition, the low-temperature conductance approaches the unitary limit. Away from the transition, for low bias voltages and temperatures, the conductance is sharply reduced. The observed behavior is compared to predictions for a two-stage Kondo effect in quantum dots coupled to single-channel leads.
\end{abstract}

DOI: $10.1103 /$ PhysRevLett.88.126803

PACS numbers: 73.23.Hk, 72.15.Qm

The observation of the Kondo effect in quantum dots $[1-3]$ has led to an increased experimental and theoretical interest in this many-body phenomenon. Unlike the conventional case of bulk metals containing magnetic impurities [4], quantum dots [5] offer the possibility to study the Kondo effect at the level of a single artificial magnetic impurity [6], allowing one to tune different parameters. Experiments on quantum dots have also revealed novel Kondo phenomena that have no analog in bulk-metal systems. In particular, multilevel Kondo effects have been studied both theoretically [7-12] and experimentally [13-15] that differ substantially from the ordinary case of a spin-1/2 Anderson impurity.

In this Letter, we present results on a strong Kondo effect in a lateral quantum dot at high magnetic field. We associate the Kondo effect with a magnetically induced crossing between a spin-singlet and a spin-triplet ground state [9-14]. In contrast to the results for a vertical semiconductor quantum dot [13] and for a carbon nanotube dot [14], we find a sharp reduction of the conductance at low bias voltage, $V_{\mathrm{SD}}$, and temperature, $T$. We ascribe the different behavior to the number of channels in the leads which couple to the states in the dot. In lateral dots, tunnel barriers are obtained by successively pinching off the propagating channels. Coulomb blockade develops when the last channel is nearly pinched off. Therefore, only one channel in each lead is coupled to the dot [16]. In vertical dots, however, the tunnel barrier characteristics are determined by the growth parameters, i.e., by the thickness of the different semiconductor materials forming the heterostructure and their relative conduction band offsets. In this case, more than one conducting channel can effectively couple to the dot states. The same is true for carbon nanotubes connected to metal leads. Our results, in combination with previous findings [13,14], show that screening of higher spin states $(S \geq 1)$ depends strongly on the number of channels coupled to the (artificial) mag- netic impurity. Comparison is made to recent theoretical studies on quantum dots in Refs. [16,17], which are partly inspired by the experimental work presented here.

Our device (Figs. 1a and 1b) consists of a lateral quantum dot [18] with a nominal diameter $\sim 300 \mathrm{~nm}$ that is further decreased by application of a negative voltage to the top gate electrode (Fig. 1a). The top gate is also used to tune the tunnel barriers between the dot and the source and drain leads. A side gate electrode is used to change the electrostatic potential on the dot, although an effect on the dot shape and tunnel barrier characteristics is unavoidable. Basic characteristics of the device are reported in Refs. $[19,20]$. All measurements have been performed in a dilution refrigerator with a base temperature of $15 \mathrm{mK}$ (effective electron temperature $\sim 40 \mathrm{mK}$ ), using a standard lock-in technique with an ac voltage between source and drain of $5 \mu \mathrm{V}$.

Figure 1c shows the linear conductance, $G$, through the dot versus side gate voltage, $V_{\mathrm{sg}}$, and perpendicular magnetic field, $B$. The $B$ dependence of the Coulomb peaks (light gray lines) is rather complicated and nonmonotonic. In some regions an enhanced valley conductance is observed. We focus on the region within the white window. Moving from right to left in this window, two Coulomb peaks approach each other and the valley conductance increases. The inset to Fig. 1c shows $G$ versus $B$ in the middle of the Coulomb valley. The highest conductance is achieved around $B=4.6 \mathrm{~T}$ and $V_{\mathrm{sg}}=-625 \mathrm{mV}$ (indicated by a "O" in Fig. 1c). This local enhancement of the valley conductance is ascribed to a singlet-triplet $(S-T)$ Kondo effect, as we substantiate below.

To illustrate the Kondo character of the enhanced valley conductance, we show in Fig. 2 the $T$ dependence of $G$ at $B=4.6 \mathrm{~T}$ (which we call $B_{0}$ ). At low $T, G$ at $V_{\text {sg }}=$ $-625 \mathrm{mV}$ approaches the unitary limit at $2 e^{2} / h$ [21-23]. Around this side gate voltage, $G$ decreases with increasing $T$. The upper curve in the inset to Fig. 2 shows the $T$ 


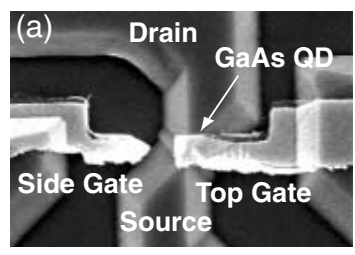

$3 \mu \mathrm{m}$

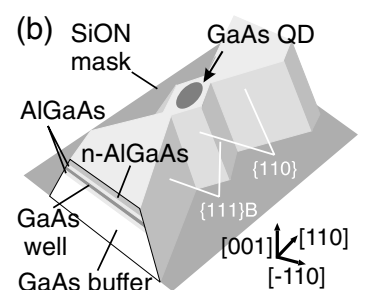

GaAs buffer [-110]

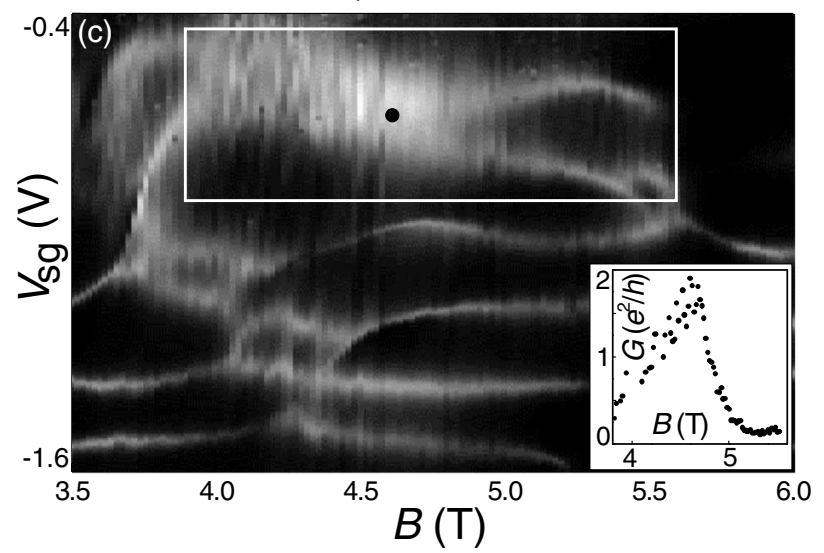

FIG. 1. (a) Scanning electron micrograph. The device consists of a dual-gated single-electron transistor fabricated by selective area growth using metal-organic vapor phase epitaxy. (b) Schematic picture of the quantum dot (QD). An $n$-doped $\mathrm{AlGaAs} / \mathrm{GaAs}$ heterostructure is selectively grown using a SiON mask, determining the dot shape. The crystal axes are indicated. The GaAs quantum well is $15 \mathrm{~nm}$ thick, lies $60 \mathrm{~nm}$ below the surface, and has an electron density of $8.7 \times 10^{15} \mathrm{~m}^{-2}$. (c) Gray-scale plot of the linear conductance, $G$, versus side gate voltage, $V_{\mathrm{sg}}$, and magnetic field, $B$. The top gate voltage is fixed at $-266 \mathrm{mV}$. Light gray lines indicate Coulomb peaks. The dark regions correspond to Coulomb blockade. The white window encloses a region of enhanced valley conductance, which is the focus of the present study. Inset: $G$ versus $B$ in the middle of the Coulomb valley within the white window.

dependence of $G$ in the middle of the Coulomb valley. The observed behavior is a typical signature of Kondo correlations. Up to our highest $T$ of $6 \mathrm{~K}$, the Kondo valley conductance keeps on decreasing, indicating an unusually strong Kondo effect. This is quite remarkable, considering the large magnetic field.

In Fig. 3a we show the $T$ dependence of the differential conductance, $d I / d V_{\mathrm{SD}}$, versus source-drain voltage, $V_{\mathrm{SD}}$, measured at $B_{0}$ and $V_{\mathrm{sg}}=-625 \mathrm{mV}$. In the ordinary, spin-1/2 Kondo effect, the Kondo resonance in the $d I / d V_{\mathrm{SD}}-V_{\mathrm{SD}}$ characteristics should split by $2|g| \mu_{B} B$ [24], where $g$ is the Lande factor and $\mu_{B}$ is the Bohr magneton. Assuming $g=-0.44$, as in bulk GaAs, this splitting is $230 \mu \mathrm{eV}$ at $B_{0}$. However, this is not observed in the $d I / d V_{\mathrm{SD}}-V_{\mathrm{SD}}$ curve at $B_{0}$ (thick solid line in Fig. 3a). Instead, we measure a single zero-bias resonance with a full width at half maximum (FWHM) of about $300 \mu \mathrm{eV}$, corresponding to a Kondo temperature $T_{K} \sim \mathrm{FWHM} / k_{B} \approx 4 \mathrm{~K}$ in the center of the Coulomb valley. This Kondo temperature is considerably larger than the values found in earlier experiments $[1-3,13,14,23]$.

Based on the above considerations, we believe that the explanation for the enhanced $G$ is an $S$ - $T$ transition around

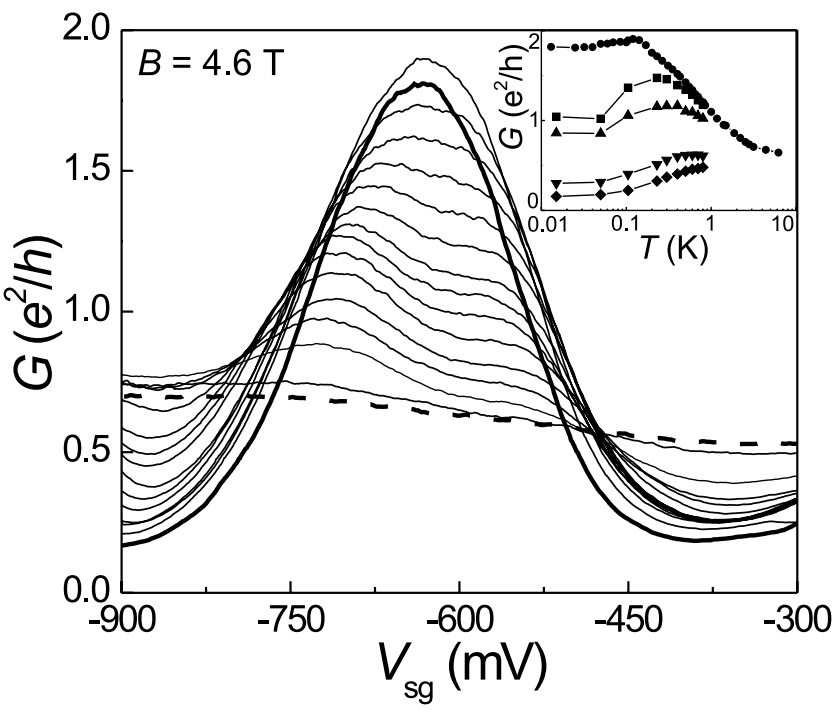

FIG. 2. $G-V_{\text {sg }}$ traces taken at $B=4.6 \mathrm{~T}$ and different temperatures, $T$, between $15 \mathrm{mK}$ (thick solid line) and $6 \mathrm{~K}$ (thick dashed line). At the lowest $T, G$ approaches the unitary limit at $2 e^{2} / h$. Inset: $G$ versus $T$ in the middle of the Coulomb valley $\left(V_{\mathrm{sg}}=-625 \mathrm{mV}\right)$ for different $B:(\boldsymbol{\square}) 4.4 \mathrm{~T}$, (О) $4.6 \mathrm{~T},(\boldsymbol{\Delta})$ $4.8 \mathrm{~T},(\boldsymbol{\nabla}) 5.0 \mathrm{~T},(\diamond) 5.2 \mathrm{~T}$. At the degeneracy field $B=B_{0}=$ $4.6 \mathrm{~T}, G$ decreases logarithmically between 0.1 and $2 \mathrm{~K}$. The curves for other $B$ show a clear bump that shifts to higher $T$ with increasing distance to $B=B_{0}$.

$B_{0}$, assuming an even number of confined electrons. As a result of the generalized Hund's rule [25], the nature of the ground state near a level crossing can change from a singlet to a triplet or vice versa. An $S$ - $T$ degeneracy leads to an enhanced low-temperature conductance as shown experimentally in Refs. $[13,14]$ and theoretically in Refs. $[9,10]$. The local conductance enhancement at $B_{0}$ in the present study suggests a similar $S-T$ Kondo effect. In the vertical quantum dot studied in Ref. [13], it is possible to determine the number of electrons in the dot and to identify the singlet and triplet states. The present data do not allow us to unambiguously identify the spin character of the states on both sides of $B_{0}$.

An interesting and unexpected feature is observed when moving away from $B_{0}$. A sharp dip develops around $V_{\mathrm{SD}}=0$ within a broader Kondo resonance. The dip vanishes upon increasing $T$, restoring the broad Kondo resonance. This is shown in Figs. $3 b-3 d$ for the representative fields $B=4.4,4.8$, and 5.0 T. The broad Kondo resonance disappears at a much slower rate with $T$ than the sharp dip, leading to the nonmonotonic behavior in the corresponding $G-T$ curves in the inset to Fig. 2. Moving away from $B_{0}$, the position of the maximum in $G$ shifts to higher $T$ and the low- $T$ conductance decreases.

Increasing $B$ from $B_{0}$, we find that the dip width grows up to $\sim 250 \mu \mathrm{V}$ (see Figs. 4a and 4b). In Fig. 4c we compare the position of the Coulomb peaks (converted from $V_{\text {sg }}$ to energy [26]) to the positions of the dip edges. We expect the on-site Coulomb interaction energy to be essentially constant within the window in Fig. 1c. Hence, we believe that the $B$ evolution of the Coulomb peak spacing 


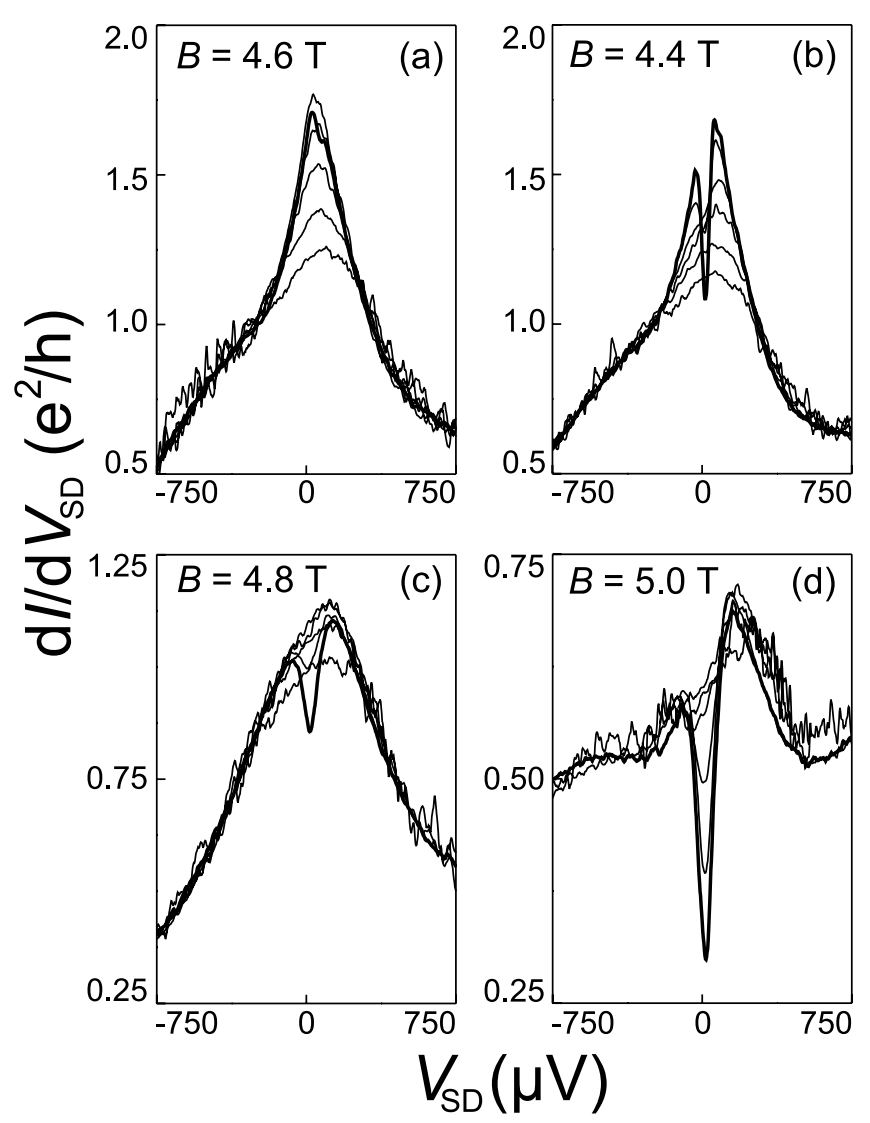

FIG. 3. (a)-(d) $T$ dependence (between $15 \mathrm{mK}$, solid line, and $800 \mathrm{mK}$ ) of the differential conductance, $d I / d V_{\mathrm{SD}}$, versus source drain voltage, $V_{\mathrm{SD}}$, in the middle of the Coulomb valley $\left[V_{\mathrm{sg}}=-625 \mathrm{mV}\right.$ (a), $-613 \mathrm{mV}$ (b), $-695 \mathrm{mV}$ (c), $-685 \mathrm{mV}$ (d)] for different $B$.

reflects the $B$ dependence of the energy difference between two consecutive levels of the dot, i.e., the $S$ - $T$ energy spacing. The dip width shows qualitatively the same $B$ dependence as the spacing between the Coulomb peaks, and thus the same as that of the $S-T$ energy spacing. The dip width at fixed $B$ is independent of $V_{\mathrm{sg}}$ within the Coulomb valley where the $d I / d V_{\mathrm{SD}}-V_{\mathrm{SD}}$ characteristics are measured (see Fig. 4c). This observation is in line with a relation between the dip width and the level spacing, irrespective of the absolute level energies.

For $3.8 \mathrm{~T}<B<B_{0}$ we find a dip whose width does not vary significantly with $B$ (see Figs. $4 \mathrm{a}$ and $4 \mathrm{~b}$ ). From Fig. 1c we note that, in the same $B$ range, the Coulomb peaks are closely and constantly spaced, denoting a virtually constant $\Delta$. This may explain why there is no clear $B$ dependence of the dip width for $B<B_{0}$.

In summary, on both sides of $B_{0}$ we observe a nonmonotonic $T$ dependence of $G$ and a narrow antiresonance superimposed on a broader Kondo resonance. The widths of these resonances correspond to two clearly distinct energy scales. Our observations are in qualitative agreement with recent theoretical predictions for quantum dots coupled to single-channel leads $[16,17]$. The single-channel character of the leads results in both studies, but under different conditions, to a two-stage Kondo effect. Two-stage
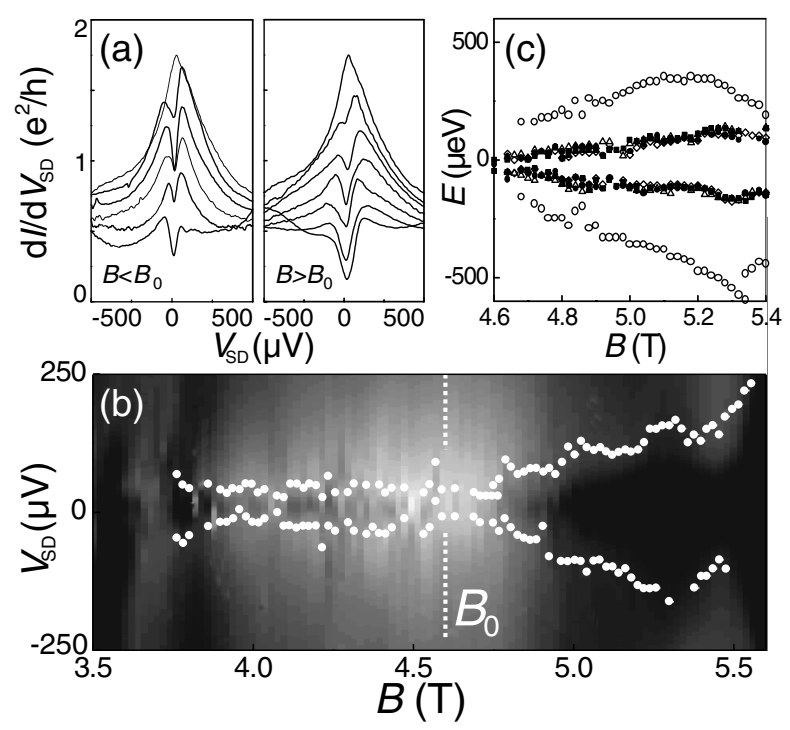

FIG. 4. (a) $d I / d V_{\mathrm{SD}}$ versus $V_{\mathrm{SD}}$ between 3.8 and $4.6 \mathrm{~T}$ (bottom to top, left), and between 4.6 and $5.2 \mathrm{~T}$ (top to bottom, right). (b) Gray-scale plot of $d I / d V_{\mathrm{SD}}$ versus $B$ and $V_{\mathrm{SD}}$. The edges of the zero-bias dip are indicated by white dots. (c) Comparison between the $B$ evolution of the dip edges [different symbols correspond to $V_{\mathrm{sg}}=-670 \mathrm{mV}(\diamond),-700 \mathrm{mV}(\circlearrowleft)$, $-730 \mathrm{mV}(\triangle)$, and $V_{\mathrm{sg}}$ in the middle of the Coulomb valley (ם)] and that of the Coulomb peaks $(\bigcirc)$. Note that the distance between the Coulomb peaks includes a (constant) charging energy.

Kondo phenomena have been theoretically studied also in other systems, such as coupled magnetic impurities in metals [27]. However, no experimental observation has been reported so far.

For a two-stage Kondo effect to occur in quantum dots, it is necessary that the dot has a spin state $S \geq 1$ coupled to single-channel leads, as illustrated in Fig. 5a for $S=1$. Instead of one low-energy scale, $T_{K}$, a two-stage Kondo effect is characterized by two separate energy scales $T_{K 1}$ and $T_{K 2}\left(T_{K 2}<T_{K 1}\right)$. The first-stage screening process with characteristic energy scale $T_{K 1}$ is an underscreened Kondo effect, reducing the net spin from $S=1$ to $S=$ $1 / 2$. The second stage of the Kondo effect, with a smaller energy scale $T_{K 2}$, reduces the spin to $S=0$, forming a spin singlet. For $T_{K 2}<\left(T, V_{\mathrm{SD}}\right)<T_{K 1}$, the first-stage Kondo screening overcomes the Coulomb blockade and $G$ is expected to reach the unitary limit. For $\left(T, V_{\mathrm{SD}}\right)<T_{K 2}$, the second stage of the Kondo effect quenches the first one, resulting in a suppression of $G$, ideally to zero. A finite electron temperature or Zeeman energy may reduce the effect of the second screening process. The signatures of a two-stage Kondo effect are a nonmonotonic $T$ dependence of $G$ and a sharp dip superimposed on the usual zero-bias resonance in the $d I / d V_{\mathrm{SD}}$, as schematically depicted in Figs. $5 b$ and $5 c$, respectively.

Our results are very similar to the schematic graphs in Figs. 5b and 5c, supporting an interpretation in terms of a two-stage Kondo effect. As the character of the ground state on both sides of $B_{0}$ may differ, the mechanism 


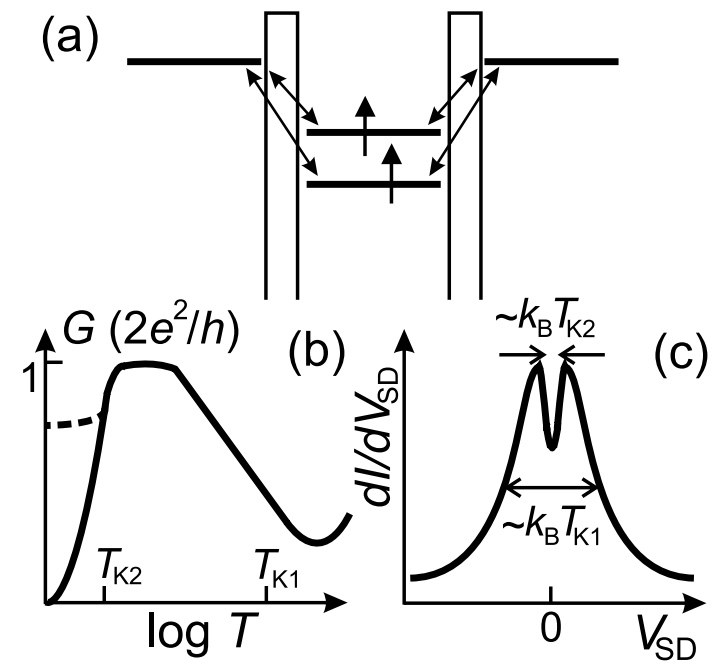

FIG. 5. Schematics of a two-stage Kondo effect. (a) Two single-particle states with total spin $S=1$ coupled to singlechannel leads. (b) $T$ dependence of $G$ showing the different regimes characterized by the energy scales $T_{K 1}$ and $T_{K 2}$. The dashed line corresponds to the case of a finite electron temperature and/or Zeeman energy. (c) $d I / d V_{\mathrm{SD}}-V_{\mathrm{SD}}$ showing a dip with a width $\sim k_{B} T_{K 2}$ within a Kondo resonance with width $\sim k_{B} T_{K 1}$.

underlying the two-stage Kondo effect need not be the same though. Hofstetter and Schoeller find a two-stage Kondo effect for a singlet ground state with a nearby triplet excited state [17]. Pustilnik and Glazman predict a two-stage Kondo effect for an $S \geq 1$ ground state when there is significant asymmetry in the dot-lead coupling [16]. The latter model, however, does not include dot excitations, which can be important in proximity of an $S-T$ transition.

For $B>B_{0}$, we can tune the $S$ - $T$ spacing, $\Delta$, between $\sim 0$ and a few hundred $\mu \mathrm{eV}$ (see the open white circles in Fig. 4c). Because of the high Kondo temperature $\left(k_{B} T_{K 1} \sim 300 \mu \mathrm{eV}\right)$, we cover the range from $\Delta \ll$ $k_{B} T_{K 1}$ to $\Delta \approx k_{B} T_{K 1}$. Over a $B$ range of about $0.5 \mathrm{~T}$ we observe that the dip width scales linearly with $\Delta$. From the observed dip width (Fig. 4c) we can estimate $T_{K 2} \lesssim 1 \mathrm{~K}$. The observed behavior for $B>B_{0}$ is in line with the results of Ref. [17] for the case of a singlet ground state with a nearby triplet. There the energy scale $T_{K 2}$ increases linearly with $\Delta$ for $\Delta \sim k_{B} T_{K 1}$. We also note that the absence of a triplet Kondo resonance for $B>B_{0}$ favors the interpretation of a singlet ground state here.

For $B<B_{0}$ there is no clear $B$ dependence of the conductance dip. Possibly, a singlet ground state is close to a triplet excited state over a relatively wide $B$ range. In this case an explanation as for $B>B_{0}$ applies. This interpretation requires a transition from a singlet ground state $\left(B<B_{0}\right)$ to a region of $S$ - $T$ degeneracy $\left(B \sim B_{0}\right)$ to again a singlet ground state $\left(B>B_{0}\right)$. Alternatively, the ground state for $B<B_{0}$ could also be a triplet, in which case the mechanism proposed in Ref. [16] may be responsible for the dip.
We conclude that, irrespective of the precise model, our results can be interpreted in terms of a two-stage Kondo effect, characterized by two well-separated energy scales.

We thank W. Hofstetter, H. Schoeller, M. Pustilnik, L. Glazman, T. Costi, W. Izumida, M. Eto, and R. Schouten for their help. We acknowledge financial support from DARPA Grant No. DAAD19-01-1-0659 of the QuIST program, the Specially Promoted Research Grant-in-Aid for Scientific Research; Ministry of Education, Culture, Sports, Science and Technology in Japan; the Dutch Organization for Fundamental Research on Matter; the Core Research for Evolutional Science and Technology (CREST-JST); and the European Union through a Training and Mobility of Researchers Program network.

[1] D. Goldhaber-Gordon et al., Nature (London) 391, 156 (1998).

[2] S. M. Cronenwett et al., Science 281, 540 (1998).

[3] J. Schmid et al., Physica (Amsterdam) 256B-258B, 182 (1998).

[4] J. Kondo, Prog. Theor. Phys. 32, 37 (1964).

[5] L. P. Kouwenhoven et al., in Mesoscopic Electron Transport, edited by L. L. Sohn et al., NATO Advanced Study Institutes, Ser. E, Vol. 345 (Kluwer, Dordrecht, Boston, 1997), pp. 105-214.

[6] L. I. Glazman and M. E. Raikh, JETP Lett. 47, 452 (1988).

[7] T. Inoshita et al., Phys. Rev. B 48, 14725 (1993).

[8] M. Pustilnik et al., Phys. Rev. Lett. 84, 1756 (2000).

[9] M. Eto and Yu. V. Nazarov, Phys. Rev. Lett. 85, 1306 (2000).

[10] M. Pustilnik and L. I. Glazman, Phys. Rev. Lett. 85, 2993 (2000).

[11] M. Pustilnik and L. I. Glazman, Phys. Rev. B 64, 045328 (2001).

[12] M. Pustilnik et al., cond-mat/0010336.

[13] S. Sasaki et al., Nature (London) 405, 764 (2000).

[14] J. Nygård et al., Nature (London) 408, 342 (2000).

[15] J. Schmid et al., Phys. Rev. Lett. 84, 5824 (2000).

[16] M. Pustilnik and L. I. Glazman, Phys. Rev. Lett. 87, 216601 (2001).

[17] W. Hofstetter and H. Schoeller, Phys. Rev. Lett. 88, 016803 (2002).

[18] F. Nakajima et al., J. Appl. Phys. 90, 2606 (2001).

[19] J. Motohisa et al., Physica E (Amsterdam) (to be published).

[20] J. Motohisa et al., Appl. Phys. Lett. (to be published).

[21] T. K. Ng and P. A. Lee, Phys. Rev. Lett. 61, 1768 (1988).

[22] A. Kawabata, J. Phys. Soc. Jpn. 60, 3222 (1991).

[23] W. G. van der Wiel et al., Science 289, 2105 (2000).

[24] Y. Meir et al., Phys. Rev. Lett. 70, 2601 (1993); N. S. Wingreen and Y. Meir, Phys. Rev. B 49, 11040 (1994).

[25] S. Tarucha et al., Phys. Rev. Lett. 84, 2485 (2000).

[26] We use $\varepsilon=\alpha V_{\mathrm{sg}}+$ constant, with $\alpha=C_{\mathrm{sg}} / C_{\Sigma}=$ $3.4 \mu \mathrm{eV} / \mathrm{mV}$. Here, $\varepsilon$ is the level energy, $C_{\text {sg }}$ is the capacitance of the side gate, and $C_{\Sigma}$ is the total capacitance of the dot.

[27] C. Jayaprakash et al., Phys. Rev. Lett. 47, 737 (1981); L. C. Andreani and H. Beck, Phys. Rev. B 48, 7322 (1993); N. Andrei and A. Jerez, Phys. Rev. Lett. 74, 4507 (1995); J. B. Silva et al., Phys. Rev. Lett. 76, 275 (1996). 